\title{
Identification and functional characterization of copy number variations in diverse chicken breeds
}

\author{
Ruili Han ${ }^{\dagger}$, Pengkun Yang ${ }^{\dagger}$, Yadong Tian, Dandan Wang, Zengxuan Zhang, Lele Wang, Zhuanjian Li, \\ Ruirui Jiang and Xiangtao Kang ${ }^{*}$
}

\begin{abstract}
Background: The detection and functional characterization of genomic structural variations are important for understanding the landscape of genetic variation in the chicken. A recently recognized aspect of genomic structural variation, called copy number variation (CNV), is gaining interest in chicken genomic studies. The aim of the present study was to investigate the pattern and functional characterization of CNVs in five characteristic chicken breeds, which will be important for future studies associating phenotype with chicken genome architecture.
\end{abstract}

Results: Using a commercial $385 \mathrm{~K}$ array-based comparative genomic hybridization (aCGH) genome array, we performed CNV discovery using 10 chicken samples from four local Chinese breeds and the French breed Houdan chicken. The female Anka broiler was used as a reference. A total of 281 copy number variation regions (CNVR) were identified, covering $12.8 \mathrm{Mb}$ of polymorphic sequences or $1.07 \%$ of the entire chicken genome. The functional annotation of CNVRs indicated that these regions completely or partially overlapped with 231 genes and 1032 quantitative traits loci, suggesting these CNVs have important functions and might be promising resources for exploring differences among various breeds. In addition, we employed quantitative PCR (qPCR) to further validate several copy number variable genes, such as prolactin receptor, endothelin 3 (EDN3), suppressor of cytokine signaling 2, CD8a molecule, with important functions, and the results suggested that EDN3 might be a molecular marker for the selection of dark skin color in poultry production. Moreover, we also identified a new CNVR (chr24: 3484617-3512275), encoding the sortilin-related receptor gene, with copy number changes in only black-bone chicken.

Conclusions: Here, we report a genome-wide analysis of the CNVs in five chicken breeds using aCGH. The association between EDN3 and melanoblast proliferation was further confirmed using $\mathrm{qPCR}$. These results provide additional information for understanding genomic variation and related phenotypic characteristics.

\section{Background}

Genetic variation occurs in many different ways, ranging from large microscopically visible chromosome anomalies to single nucleotide changes. The subset of potential genetic variations, deletions, insertions, duplications, and complex multi-site variants, is collectively referred to as copy number variants (CNVs) [1]. CNVs range from approximately $50 \mathrm{bp}$ to several $\mathrm{Mb}$ in size [2-4] and might exhibit potentially larger effects through the disruption of genes and alteration of gene dosages, disruption of

\footnotetext{
* Correspondence: xtkang2001@263.net

'Equal contributors

College of Animal Science and Veterinary Medicine, Henan Agricultural University, Henan Innovative Engineering Research Center of Poultry Germplasm Resources, Zhengzhou, Henan 450002, China
}

coding sequences and perturbation of long-range gene regulation [5]. In the last decade, many studies have been conducted on the distribution, function, and role of CNVs in diseases involving DNA segments in the human genome [2,3,5-15]. Recently, genome-wide CNVs have not only been identified in humans but also in domestic animals, such as cattle [8,16-19], sheep [20], goat [21], pig [22-25], and poultry [26-35].

China has a wide variety of indigenous chicken breeds, and chicken genomics is likely to have major applications and benefits in agriculture, comparative genomics, evolutionary biology, systematics, and models of development and human disease [26]. Previous studies have reported that CNVs are responsible for the phenotypic changes in chicken. Examples of phenotypes associated 
with CNVs in the chicken include late feathering on chromosome Z (GGAZ) [36], pea comb on GGA1 [37], dark brown plumage color on GGA1 [38], and dermal hyperpigmentation on GGA20 [39]. Furthermore, there is a large genetic distance between Chinese and European chicken populations, which facilitates the detection of fruitful breed-specific CNVs. However, compared with humans and other model organisms, there is limited research on the extent and impact of CNVs in the chicken genome. The aim of the present study was to investigate the extent and pattern of CNVs in five characteristic chicken breeds. The CNVs detected herein are complementary to the CNV map in the chicken genome, and this information will be important for future studies associating phenotype with genome architecture.

\section{Results}

Genome-wide detection of CNVs

Using a commercial $385 \mathrm{~K}$ array-based comparative genomic hybridization $(\mathrm{aCGH})$ genome array (Roche NimbleGen, Inc., Madison, WI, USA), we performed CNV discovery using 10 chicken samples from four Chinese local breeds including Xichuan Black-bone chicken (XC), Silkie chicken (SK), Lushi chicken (LS), and Gushi chicken (GS), and one French breed Houdan chicken (HD) (Figure 1). Based on the WUGSC2.1/galGal3 genome sequence, a total of $1743 \mathrm{CNVs}$ were identified in the genomes of these chicken breeds. After eliminating probes with uncertain chromosomal loci, we identified 446 non-redundant CNVs for the chicken autosome GGA1-28 and the $\mathrm{Z}$ sex chromosome. The mean and median lengths of the CNVs were 45.4 and $25.0 \mathrm{~kb}$, respectively, ranging from $9.6 \mathrm{~kb}$ to $1.5 \mathrm{Mb}$ in length. Among these segments,
171 CNVs involved a DNA sequence gain, while 275 CNVs involved a DNA sequence loss. The total number of CNVs for each breed was 91 in XC, 74 in SK, 81 in LS, 111 in GS, and 89 in HD (Additional file 1).

After aggregating the overlapping CNVs, a total of 281 CNVRs across the WUGSC2.1/galGal3 genome sequence were identified, covering $12.8 \mathrm{Mb}$ of the chicken genome and $1.07 \%$ of the entire chicken genome. The mean and median sizes of the CNVRs were 45.6 and $25.0 \mathrm{~kb}$, respectively. Most of the CNVRs (76.5\%) were detected in a single individual, while the remaining CNVRs (33.5\%) were identified in more than one individual (Additional file 1). We identified only one CNVR (chr11: 14877827-14917568) present in all the ten individuals tested. Compared with previous studies in the chicken [28,29,31-34], 114 (23.13\%) CNVRs overlapped with previously reported CNVRs (Table 1), while 216 (76.87\%) CNVRs were reported for the first time. Among the 114 overlapping CNVRs, 49 CNVRs have been identified in all studies, and 65 CNVRs have been reported in various studies (Additional file 2).

The 281 CNVRs identified were present on all chicken GGA1-28 autosomes and sex chromosome Z. A total of 159 (57\%) CNVRs were located on chromosome 1-6, chromosome 10 and sex chromosome Z, while fewer CNVRs were located on chromosome 16 and chromosome 23. Concerning copy number status, 181 (64\%) CNVRs involved a sequence loss, 91 (32\%) CNVRs involved a sequence gain, and the remaining 9 (3\%) CNVRs involved both a sequence loss and a sequence gain within the same region (Figure 2).

After converting the location of the probes from galGal3 to galGal4 (2011 CGSC Gallus gallue-4.0/galGal4) according to the probe sequence, 309 non-redundant CNVs were

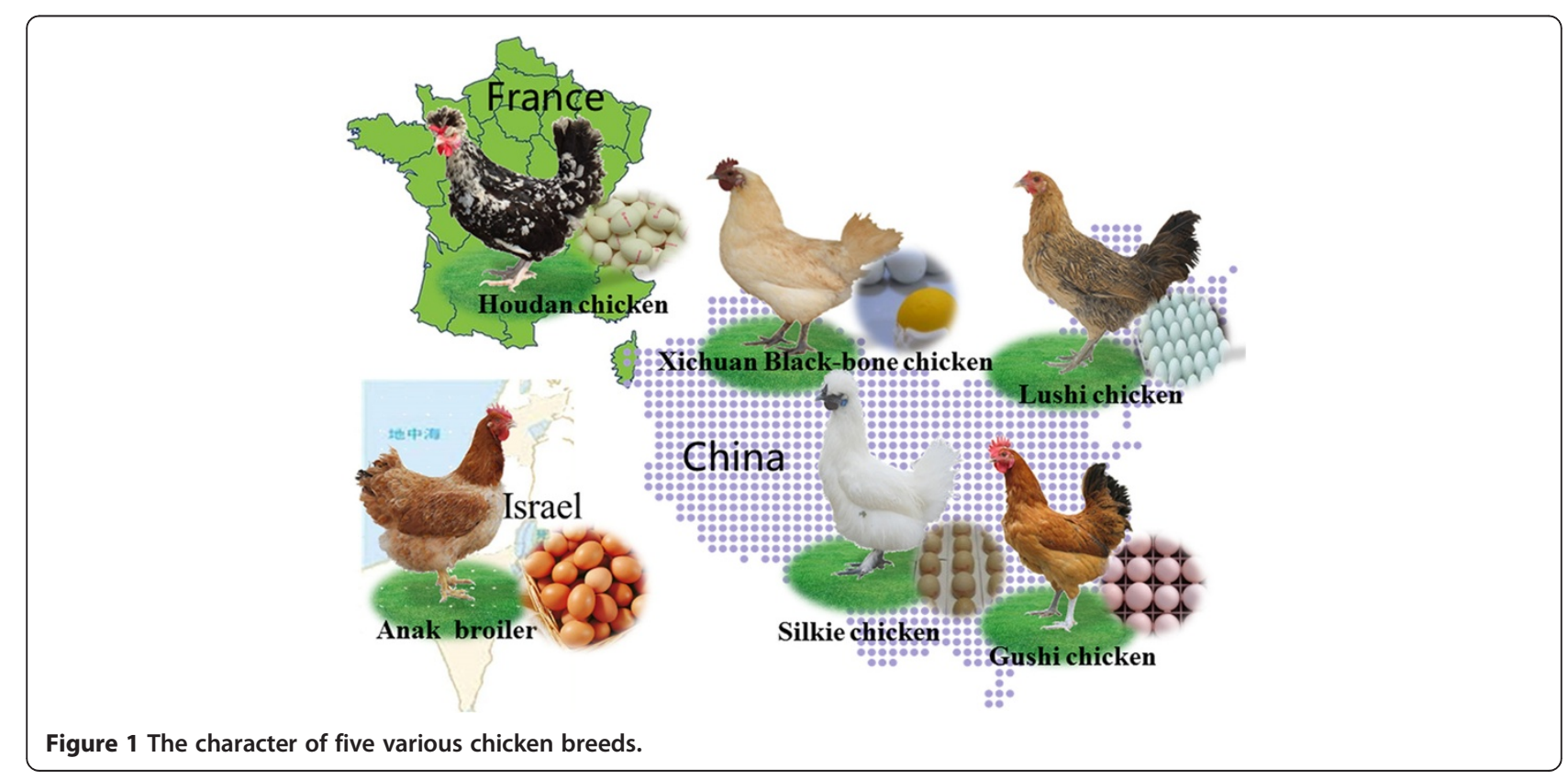


Table 1 Comparison of chicken CNVRs identified in this study and in previous studies using 385 K NimbleGen whole genome-tiling arrays

\begin{tabular}{|c|c|c|c|c|c|c|c|c|c|c|c|c|}
\hline \multirow[t]{2}{*}{ Study } & \multicolumn{8}{|c|}{ Chicken CNVRs identified in this study and in previous studies } & \multicolumn{4}{|c|}{ Overlaps with this study } \\
\hline & Platform & Samples & $\begin{array}{l}\text { Breeds/ } \\
\text { Species }\end{array}$ & CNVR & Range (kb) & $\begin{array}{l}\text { Median } \\
\text { (kb) }\end{array}$ & $\begin{array}{c}\text { Mean } \\
(\mathrm{kb})\end{array}$ & $\begin{array}{c}\text { Total } \\
\text { length } \\
\text { (Mb) }\end{array}$ & Count* & Percentage & $\begin{array}{l}\text { Total } \\
\text { length } \\
\text { (kb) }\end{array}$ & Percentage \\
\hline Griffin et al. [34] & $\begin{array}{c}385 \text { k } \\
\text { NimbleGen }\end{array}$ & 2 & 2 & 21 & 18.77-900.00 & 90.00 & 141.83 & 2.84 & 6 & 2.14 & 418.06 & 3.27 \\
\hline Völker et al. [29] & $\begin{array}{l}385 \text { k } \\
\text { NimbleGen }\end{array}$ & 1 & 2 & 27 & $20.15-950.00$ & 127.10 & 211.77 & 5.29 & 3 & 1.07 & 710.00 & 5.55 \\
\hline Wang et al. [32] & $\begin{array}{c}385 \text { k } \\
\text { NimbleGen }\end{array}$ & 10 & 3 & 91 & 10.30-2030.06 & 42.59 & 157.17 & 15.72 & 14 & 4.98 & 814.91 & 6.37 \\
\hline Wang et al. [31] & 400 k Agilent & 6 & 3 & 130 & $6.20-649.12$ & 14.43 & 25.70 & 3.34 & 12 & 4.27 & 339.79 & 2.65 \\
\hline Tian et al. [28] & 400 k Agilent & 22 & 11 & 308 & $5.82-2025.34$ & 14.6 & 35.1 & 10.8 & 29 & 10.32 & 818.53 & 6.39 \\
\hline $\begin{array}{l}\text { Crooijmans et al. } \\
{[33]}\end{array}$ & Agilent244K & 64 & 15 & 1556 & $4.87-4365.80$ & 26.0 & 45.2 & 70.1 & 50 & 17.79 & 2151.12 & 16.81 \\
\hline This study & $\begin{array}{c}385 \text { k } \\
\text { NimbleGen }\end{array}$ & 10 & 5 & 281 & $9.63-1522.58$ & 25.0 & 45.6 & 12.8 & - & - & - & - \\
\hline
\end{tabular}

*:The number of CNVRs identified in the present study that have been previously reported.

identified in the five chicken breeds. The mean and median lengths of the CNVs were 40.7 and $22.5 \mathrm{~kb}$, respectively. A total of $138 \mathrm{CNVs}$ involved a DNA sequence gain, while $171 \mathrm{CNVs}$ involved a DNA sequence loss. The lengths of the CNVs ranged from 1.6 to $320.5 \mathrm{~kb}$. The total number of CNVs for each breed was 67 in XC, 66 in SK, 63 in LS, 72 in GS, and 41 in HD (Additional file 1). A total of 192 CNVRs were identified based on the galGal4 genome sequence. The length of these CNVRs ranged from 8.65 to $320 \mathrm{~kb}$, with mean or median lengths of 36.1 and $22.5 \mathrm{~kb}$, respectively. Concerning copy number status, 112 (58\%) CNVRs involved a sequence loss, 75 (39\%) CNVRs involved a sequence gain, and the remaining 5 (3\%) CNVRs involved both variations (Additional file 1).

\section{Gene contents of the chicken CNV regions}

Using the BioMart (http://www.biomart.org/) data management system, we retrieved the gene content of the

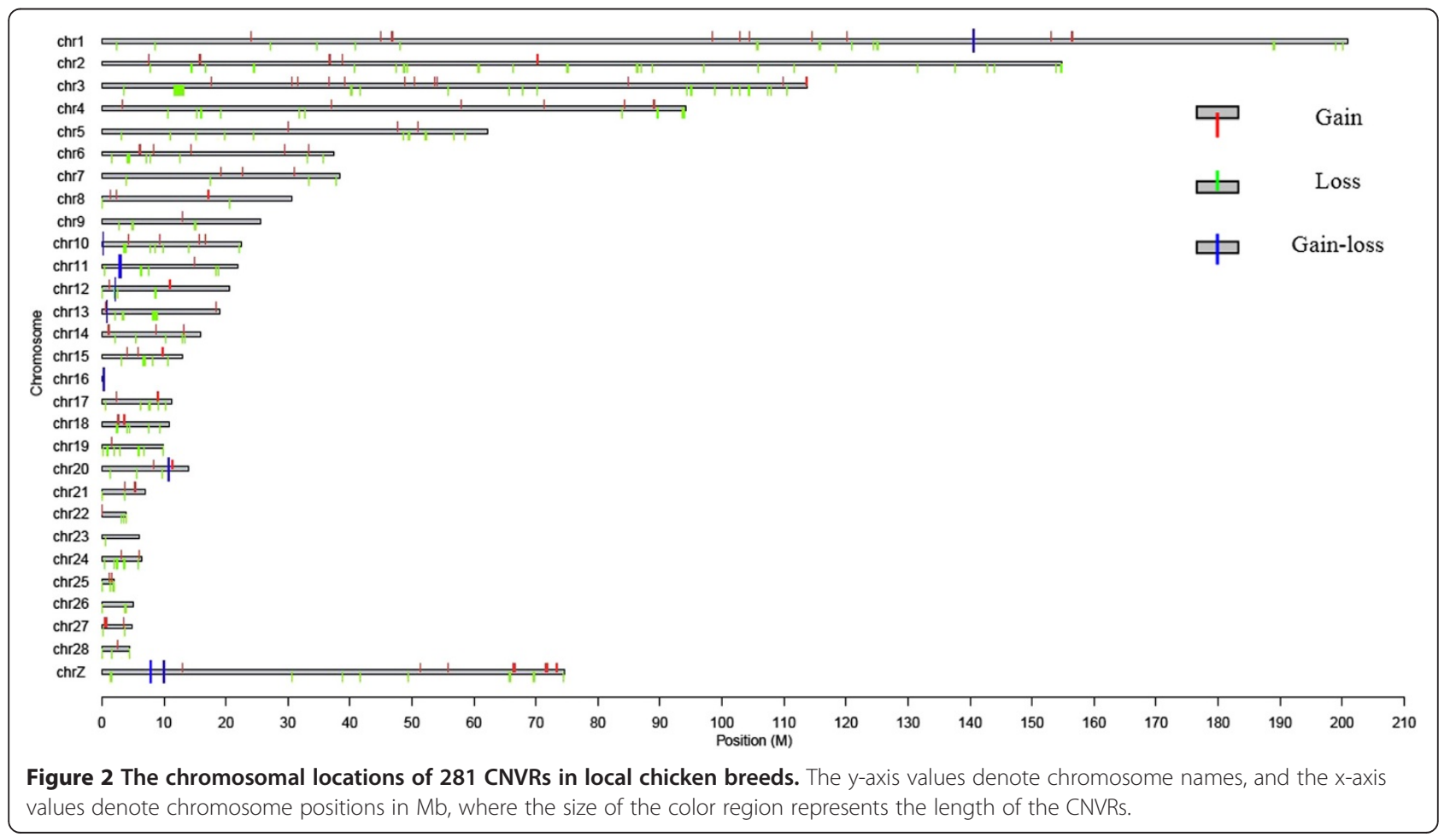


CNVRs. A total of 231 genes (Additional file 3) completely or partially overlapped with previously identified CNVRs, including 221 protein-coding genes, 1 pseudo gene, 8 miRNA genes, and 1 rRNA gene. These genes were distributed among 167 of the 192 CNVRs identified, while the other CNVRs did not contain any annotated genes.

The Gene Ontology (GO) analyses revealed 87 GO terms and the Kyoto Encyclopedia of Genes and Genomes (KEGG) pathway displayed three pathways (Additional file 4). Among the $87 \mathrm{GO}$ terms, four terms were statistically significant after Benjamini correction. The significant GO terms primarily involved cell adhesion, transcription factor activity, sequence-specific DNA binding, and transcription regulator activity $(P<0.05)$. The analyses also revealed some enriched terms with marginal significance, involved in biological adhesion, pattern specification, embryonic morphogenesis, appendage development and limb development $(P<0.1)$. The KEGG pathway analyses indicated that the genes in the CNVRs were enriched in three pathways, involving cell adhesion molecules, hematopoietic cell lineage, and leukocyte transendothelial migration, however, these terms were not statistically significant after Benjamini correction.

Next, we analyzed whether our CNVRs mapped to known QTLs in the chicken QTLdb [Release 20 (Apr 20, 2013): http://cn.animalgenome.org/cgi-bin/QTLdb/ $\mathrm{GG} /$ index]. Because the confidence intervals for some QTLs were too large, we focused on QTLs with confidence intervals less than $10 \mathrm{Mb}$. A comparison of the overlapping CNVRs with QTLs revealed a total of 143 QTLs in 83 CNVRs, affecting a wide range of traits, including body weight, body size, carcass traits, fatness traits, Marek's disease-related traits, and reproductive traits (Additional file 5).

\section{Quantitative PCR analysis of selected CNV regions}

Quantitative PCR (qPCR) was performed to validate the aCGH data at six CNVR loci (Figure 3). Two (THRSP and $P C C A$ ) of the six loci served as references for no variation in copy number, while four loci (chr1: 44748534-44773370, chr4: 85228947-85423958, chr20: 11111788-11248088, and chrZ: 10472242-10661362) were CNVRs detected using an aCGH genome array in five chicken breeds. The qPCR results indicated that all four $\mathrm{CNV}$ loci had greater variance than the references (Figure 3), suggesting that the four loci were truly CNVs.

The qPCR results for the THRSP locus showed minimal variations among 25 birds (Figure 3A). We attributed these variations to random errors, including DNA dilution errors. Similar qPCR results were obtained for the PCCA locus in 25 birds, which also showed minimal variations among birds (Figure $3 \mathrm{~B}$ ).
In the present study, the aCGH analysis revealed the PRLR locus in $2 \mathrm{XC}, 2 \mathrm{HD}, 1 \mathrm{LS}$, and 1 GS. Subsequent qPCR analyses revealed a single copy in SK, while GS, LS, and XC had variable copy numbers, ranging from 1 to 4 copies. The results showed that female birds had 1 to 3 copies and males had 2 to 6 copies in HD (Figure 3C).

Moreover, the aCGH assay identified the SOCS2 locus in three birds, involving a gain of copy variation. The qPCR analysis showed that the variation in copy number was far more frequent (Figure 3D) than that identified in the aCGH. GS, LS, and SK had a single copy, while HD and $\mathrm{XC}$ had variable copy numbers, ranging from 1 to 5 copies.

The aCGH assay revealed RHACD8 locus in $2 \mathrm{LS}, 2$ GS, 1 GF and 1 SK. The qPCR data indicated that the relative copy number for the RHACD 8 locus was highly variable among chickens. The birds with the highest copy numbers had five times as many copies as those with the lowest copy numbers. The results of the aCGH array and qPCR analyses suggested that this CNVR locus was a common variation in the autosomal region, without breed specificity (Figure 3E).

A specific duplication of a CNVR occurred on chromosome 20at base pair positions 1111178811248088 and 11654170-11820202 encoding six annotated functional genes including the EDN3 gene. The aCGH assay revealed two loci in $2 \mathrm{SK}$ and $2 \mathrm{XC}$. The qPCR results showed that GS, LS, and HD had a single copy number, and the average copy number for SK and $\mathrm{XC}$ was approximately 2 and 1.5-fold, respectively, higher than that of these three breeds (Figure $3 \mathrm{~F}$ ). To confirm this result, we examined the copy numbers of 14 chickens (7 SK and 7 XC) (Figure 4). Similar qPCR results were obtained for the EDN3 locus. To determine the copy numbers in heterozygotes (Fmfm), the genomic copy number was estimated using qPCR for three genotypes. The qPCR analysis confirmed the duplication of the EDN3 loci in the FmFm genotype with an estimated copy number of approximately 2 -fold that of wild-type individuals (fmfm genotype), while the heterozygous genotype (Fmfm) likely involved a 1.5-fold duplication (Figure 5).

\section{Discussion}

Local Chinese chicken breeds are considered important genetic resources because of their high product quality, favorable flavor, and strong disease resistance. Here, using a tiling oligonucleotide aCGH approach, we reported the CNV survey among five unrelated characteristic chicken breeds. The number of non-redundant CNVs and CNVRs in the WUGSC2.1/galGal3 genome sequence was 446 and 281, respectively, and 309 and 192, respectively, in galGal4. Both the numbers of non- 


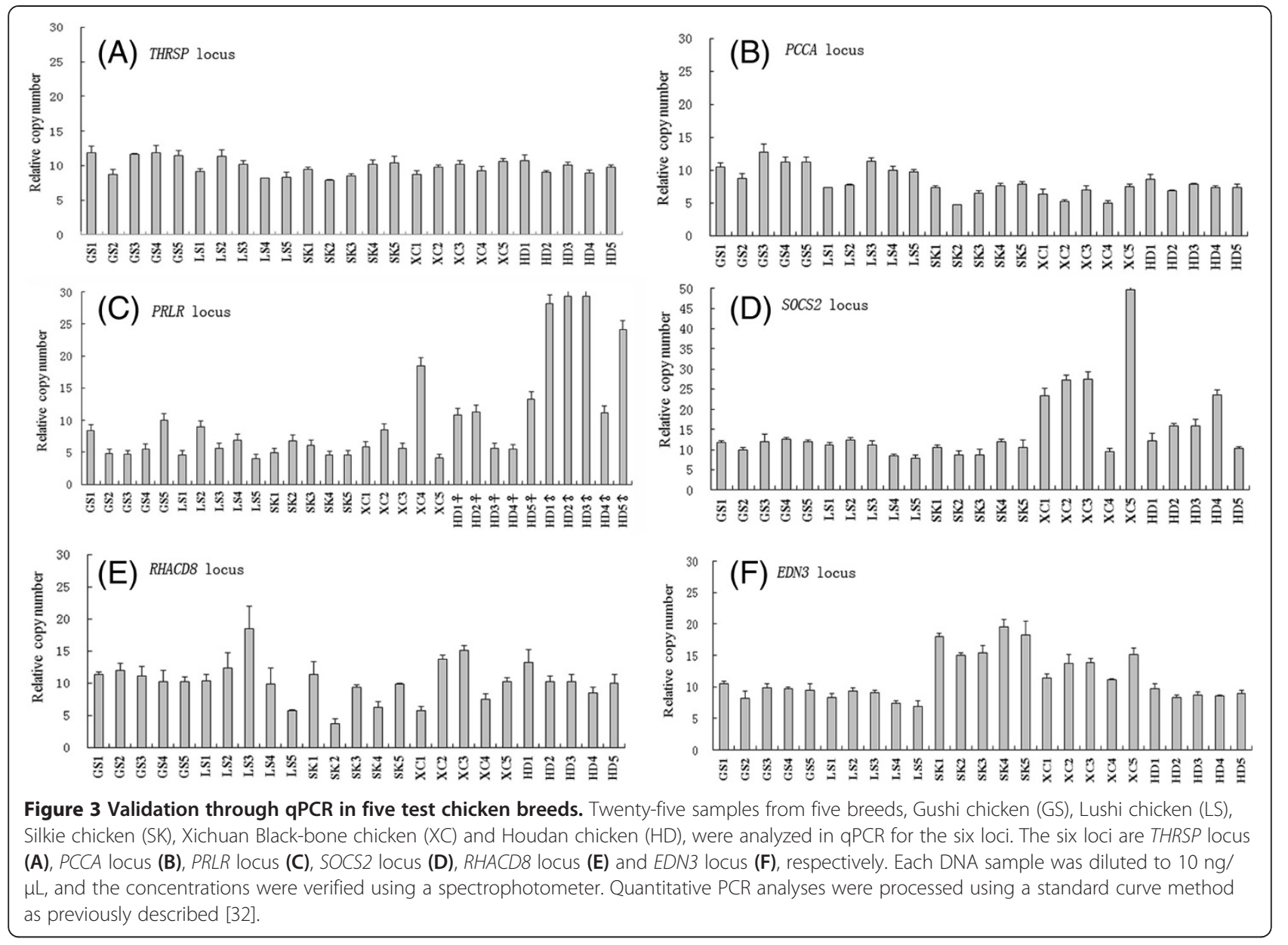

redundant CNVs and CNVRs were less in galGal4 than in galGal3 because some galGal3 probe sequences could not be successfully converted to galGal4 probe sequences. The number of CNVs and CNVRs reported were much fewer than those identified in mammals and humans.

A comparison of the CNVRs in the five breeds indicated fewer CNVs and CNVRs in HD than in the other four chicken breeds in China, and CNVR-gains were more frequent than CNVR-losses (43 losses, 46 gains) in HD. This observation might reflect the fact that the HD is different from the other four local Chinese chicken breeds in origin, appearance, and production performance.

The estimated cumulative CNVR length of $12.8 \mathrm{Mb}$ (1.1\% of the genome) was relatively long compared with that reported in recent studies [23,24,27,34], but lower expected when considering the sample size. Indeed, Crooijmans et al. [33] reported that CNVRs represented almost $5.4 \%$ of the chicken genome when samples from 64 animals were used for testing. This difference might reflect the limited CNV coverage of the platform used in the present study, resulting in a significant underestimation of real CNVs in chickens because a limited number of individuals were surveyed. Thus, a greater number of birds should be examined to obtain a comprehensive picture of chicken CNVs. Furthermore, the incompleteness of the chicken genome assembly, suggests that a significant portion of the genome was not surveyed. The entire W chromosome was excluded from the analysis, and all probes assigned to ChrUn and other random chromosomes were also excluded.

To retrieve the information and annotation for the CNVRs based on the newest chicken genome sequence, distinct from previous studies on the gene contents of chicken CNVRs, we converted the location of the probes from galGal3 to galGal4 (2011 CGSC Gallus gallue-4.0/ galGal4) according to the probe sequence, and the gene contents were processed using the galGal4 genome. The gene content analysis detected 231 Ensembl genes among the 192 identified CNVRs. Among these, 167 protein-coding genes, such as PRLR and MTAP of chrZ, RHACD8 of chr4, SLMO2, TUBB1, and EDN3 of chr20, etc., were annotated and reported in previous studies [32,33]. Notably, two CNVRs (chr20: 11111788-11248088 and chr20: 11654170-11820202) were identified on chromosome 20. The distance between the loci was 


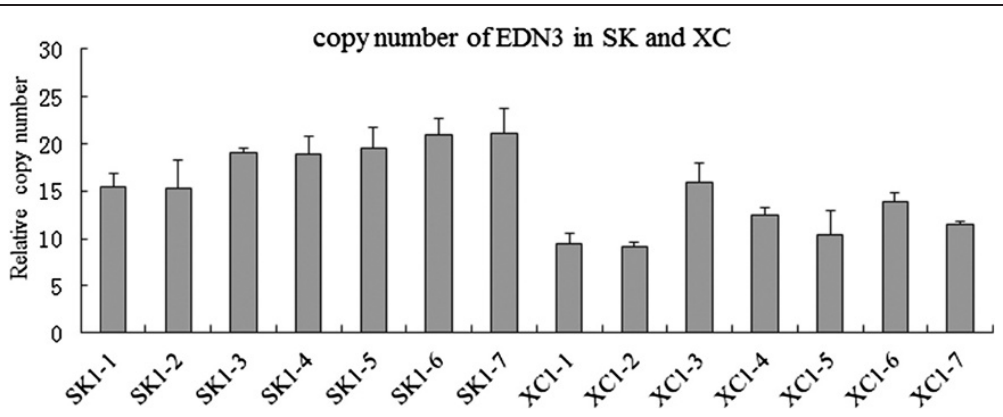

Figure 4 Validation through qPCR for the EDN3 loci in Silkie and Xichuan Black-bone chickens. Fourteen samples from two breeds, Silkie chicken (SK) and Xichuan Black-bone chicken (XC), were analyzed using qPCR for the EDN3 locus. Each DNA sample was diluted to $10 \mathrm{ng} / \mu \mathrm{L}$, and the concentrations were verified using a spectrophotometer. QPCR analyses were processed using the same standard curve method as applied in the validation test.

419.6 kb. The CNVR chr20: 11111788-11248088 has been associated with dermal hyperpigmentation in chickens [39]. In the present study, six annotated functional genes encoding $E D N 3$, zinc finger protein 831 (ZNF831), slowmo homolog 2 (SLMO2), tubulin beta-1 (TUBB1), cathepsin Z (CTSZ), partial TH1-like (Drosophila) $(T H 1 L)$, and one miRNA were identified in this CNVR. Consistently, an association between EDN3 and melanoblast proliferation has been previously reported in chickens [39-41].

In addition, we also identified a new CNVR involving a sequence loss of $27.7 \mathrm{~kb}$ in the SK (chr24: 34846173512275). This locus contains the gene encoding sortilinrelated receptor (SORL1) [ENSGALG00000006598]. The SORL1 protein belongs to a super family of low-density lipoprotein receptors that bind apolipoprotein $\mathrm{E}$ and have been implicated in cholesterol metabolism [42]. In a previous study, we showed that the contents of cholesterol in Silkie eggs was higher than that in Lohmann and Lushi eggs [43], suggesting a correlation between the cholesterol content and the loss of $27.7 \mathrm{~kb}$ of the SORL1 gene. Therefore, this CNVR is worth further investigation.

The data obtained from GO term and pathway analyses and an examination of overlapping QTLs in the chicken QTLdb suggested that these genes function in numerous molecular functions, indicating the potential of these genes as promising resources for exploring the genetic basis of phenotypic variation within and among breeds. Specifically, 83 CNVRs partially or completely overlap with 143 QTLs, and these QTLs are involved in many important traits in chickens, including growth traits, carcass traits, meat quality traits, reproductive traits, and disease-related traits. Thus, the CNVRs identified herein might represent valuable resources for studying Chinese chicken genome diversity, including the structural variation mechanisms associated with chicken traits. Together with SNPs, CNVs will be an important complement to molecular marker and genome-

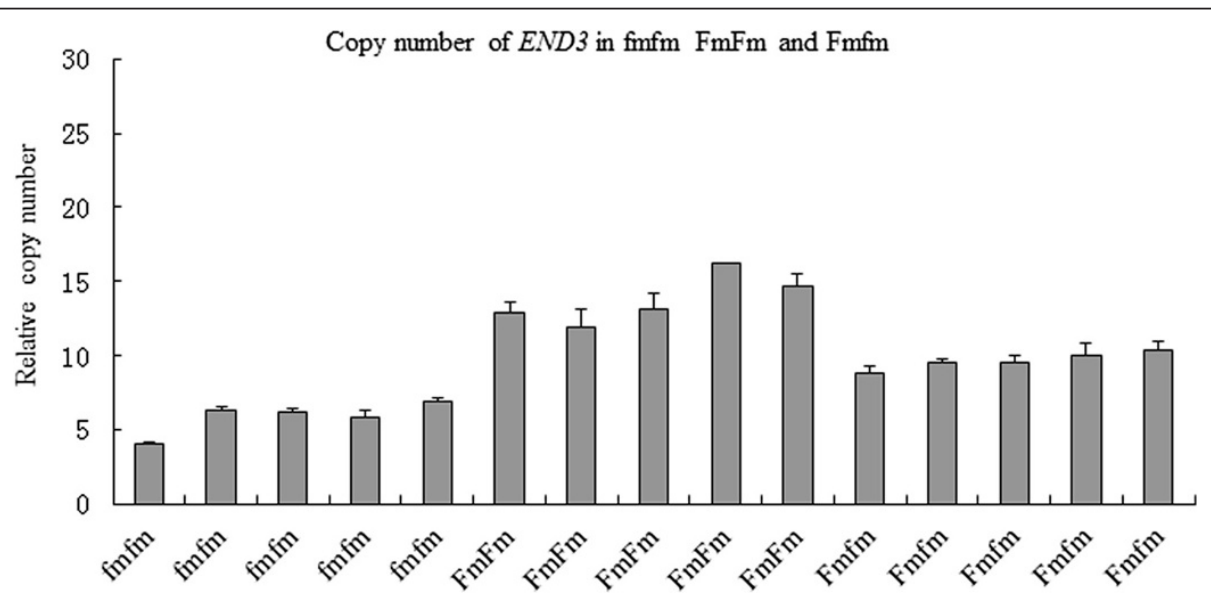

Figure 5 Quantitative PCR analysis confirmed the duplication of the EDN3 loci in three different genotypes. Fifteen samples for the three genotypes (FmFm, fmfm, and Fmfm) were analyzed using qPCR for the EDN3 locus. Each DNA sample was diluted to $10 \mathrm{ng} / \mathrm{\mu L}$, and the concentrations were verified using a spectrophotometer. The results of the GPCR analyses were processed using the same standard curve method as performed in the validation test. 
wide association studies, and these variations might account for some of the missing heritability of complex traits [44].

The presence of two copies of the PRLR locus has been associated with the slow feathering phenotype, and the lack of one copy has been associated with the early feathering phenotype [36]. Subsequent qPCR analyses of 20 female birds showed that a single copy of this gene in SK, 1 to 2 copies in GS and LS, and 1 to 4 copies in XC. The female SK exhibited the slow feathering phenotype and the female XC, LS and GS showed both slow and fast feathering phenotypes. Moreover, in HD, the results showed that female birds have 1 to 3 copies and male birds have 2 to 6 copies. We propose that the differences in the copy numbers might reflect a gain of 2 PRLR loci in HD. Thus, we provide the first report that a gain of 2 $P R L R$ loci also induced late feathering in HD. However, further studies are needed to examine the reliability of this finding. The PRLP locus is located on the $\mathrm{Z}$ chromosome, and because homozygote (ZZ) individuals are male and heterozygote ( $\mathrm{ZW})$ individuals are female, these results showed that the copy number of male birds is twice that of female birds. The results of a separate qPCR assay in HD were consistent with this idea. A sexlinked late feathering allele $\mathrm{K}$ containing 2 copies of the $P R L R$ locus has been introduced into commercial flocks and used widely for sexing hatchlings. This $\mathrm{K}$ allele is incompletely dominant to the early feathering $\mathrm{k}^{+}$allele containing one copy of the PRLR locus. Suppliers typically cross $\mathrm{k}^{+} \mathrm{k}^{+}$males with KW females to generate female progeny with the early feathering phenotype $\mathrm{k}^{+} \mathrm{W}$ containing one copy of PRLR, while the male progeny have a late feathering phenotype $\mathrm{Kk}^{+}$containing three copies of PRLR.

We observed that SK and XC were the only two breeds to show CNVs at the EDN3 locus. Dorshorst et al. [40] reported that Fm results from an inverted duplication of two genomic regions, each greater than $100 \mathrm{~kb}$, located on chromosome 20, resulting in the increased expression of the EDN3 gene. We concluded that the dermal hyperpigmentation of these two Chinese local chicken breeds also resulted from a CNV in this region. SK and XC are closely distributed in southeast China, likely reflecting the fact that these two breeds originated from the same place or have traits that were purposely bred into different strains. To explain the observation that the copy number in SK was higher than that in $\mathrm{XC}$, we propose that both heterozygotes $(\mathrm{Fmfm})$ and homozygotes $(\mathrm{fmfm})$ are present in XC. Using qPCR analysis, Dorshorst et al. [40] confirmed the duplication of both genomic regions in Fm birds, with an estimated copy number of approximately 1.5-2-fold that of wild-type individuals, suggesting that some Fm birds were likely heterozygous for a mutant allele comprising a 2-fold duplication. This result suggests that this locus might be a molecular marker for the selection of dark skin color in poultry production.

\section{Conclusions}

Here, we reported a genome-wide analysis of CNVs in five chicken breeds using an aCGH array. The mapping of CNVs will contribute to association studies of economic performance. Among the four CNVRs selected for validation through qPCR, all four variations exhibited the expected copy number differences. The association between EDN3 and melanoblast proliferation was further confirmed in the present study. Additional, in the present study, we report a new CNVR (chr24: 3484617-3512275), encoding SORL1 gene, with a copy number change only in black-bone chickens. These results provide a preliminary foundation for investigating the association between various phenotypes and CNVs. Future studies are required to assess the functional significance of $\mathrm{CNVs}$ and determine the impact of these variations on economic traits in chicken.

\section{Methods}

\section{Ethics statements}

The entire procedure for the collection of blood samples of the all animals was performed in strict accordance with the protocol approved by the Institutional Animal Care and Use Committee (IACUC) of Henan Agricultural University.

\section{Sample preparation}

In the present study, 10 individuals were selected from five chicken breeds (two females from each breed) and used as test samples, while one female Anka broiler was used as a reference. The five chicken breeds included four Chinese indigenous breeds, Xichuan Black-bone chicken (XC), Silkie chicken (SK), Lushi chicken (LS), Gushi chicken (GS) and one French commercial breed, Houdan chicken (HD) (Figure 1).

All blood samples used in the present study were collected in $0.5 \mathrm{M}$ EDTA and stored at $-20^{\circ} \mathrm{C}$ until further DNA isolation. The DNA was isolated using the DNeasy Blood and Tissue Kit (Qiagen, Hilden, Germany). The concentration and quality of the DNA samples were quantified using a NanoDrop (NanoDrop Technologies, Wilmington, DE, USA) and 1\% agarose gel electrophoresis.

Ten samples (two from each bird) were analyzed using an aCGH array and fifty-nine samples were analyzed through qPCR. Twenty-five samples (five from each breed) were analyzed for all the six loci. In addition, another five male HD were analyzed for the PRLR locus, and twenty-nine samples (7 SK and 7 XC; 5 SK, 5 Hisex Brown A line and 5 first filial generations of them) were analyzed for the EDN3 locus. 


\section{Oligonucleotide array CGH}

NimbleGen chicken whole genome tiling array (Catalog Number/Design Name B3791001-00-01, galGal3_ WG_CGH - Roche NimbleGen, Milton Keynes, UK) was used for all microarray experiments. This array contains 385,000 oligonucleotide probes (http://www. nimblegen.com) with a median probe spacing of 2,586 bp. Genomic DNA labeling, hybridization and array scanning were performed according to the manufacturer's instructions. Each test DNA sample (labeled with $\mathrm{Cy} 3$ ) was cohybridized with the reference female broiler sample (labeled with Cy5). The initial data analysis (normalization and segmentation) was performed using DEVA 1.2 software (Roche-NimbleGen). Next, each WUGSC2.1/galGal3 probe sequence was converted to galGal4 genome sequences, followed by analysis using NimbleScan 2.4 software (segMNT algorithm). The technical specifics for NimbleGen software are provided at http://www.nimblegen.com/ products/lit/lit.html.

\section{Identification of CNVs and CNVR}

The high confidence calls were obtained according to Wang et al. [32], namely segments of five or more probes with mean $\log _{2}$ ratio shift from baseline greater than $+/-0.3$ were flagged as candidate CNVs. Probes from uncertain chromosomal loci (Chr\#-random, ChrUnrandom, chrE22C19W28_E50C23, chrE64, and W chromosome in the UCSC database) were removed from the results. The CNVRs were determined after aggregating the overlapping CNVs identified across all samples according to Redon et al. [2]. We used the standards (overlap $\geq 1 \mathrm{bp}$ ) to evaluate overlaps between the results obtained in the present study and those of previous reports. In the present study, the range of overlap is 3.7-332.7 kb. Raw aCGH data for this study have been deposited in GenBank GEO database under accession number: GSE49889, http://www.ncbi.nlm. nih.gov/geo/query/acc.cgi?acc=GSE49889.

As an outdated version of this software was used in the present study (source - UCSC, build - galGal3), we first converted the galGal3 probes to galGal4 probes and subsequently performed the CNV analysis using a process similar to that described above. The functional annotation and analysis of the CNVR were based on the converted CNVRs.

\section{Functional annotation and analysis of the CNVR}

To provide insight into the functional enrichment of the CNVs, a functional annotation was performed using the DAVID Bioinformatics Resources software, version 6.7 (http://david.abcc.ncifcrf.gov/summary.jsp) to obtain GO terms [45] and KEGG [46] pathway analyses. Because only a limited number of genes in the chicken genome have been annotated, we converted the chicken Ensembl gene IDs to orthologous human Ensembl gene IDs using BioMart software, and the statistical significance was using the modified Fisher's exact test and Benjamini correction for multiple testing. We also performed an overlap analyses between the CNVRs identified and the reported QTL regions annotated in the chicken QTL database (April 20, 2013, (http://www.animalgenome. org/cgi-bin/QTLdb/GG/index).

\section{CNV validation by quantitative PCR}

Quantitative PCR primers for EDN3 were designed using Primer 5.0 software. The primers for SOCS2, THRSP, PCCA, RHACD 8 and PRLR were designed as previously described [31,32]. The primer sequences are listed in Additional file 6.

The qPCR assays conducted using SYBR SELECT MASTER MIX (Life Technologies, California, USA). The reactions were performed in $20 \mu \mathrm{l}$ containing $20 \mathrm{ng}$ of genomic DNA and $0.5 \mu \mathrm{M}$ of each primer. The cycling conditions included pre-incubation at $50^{\circ} \mathrm{C}$ for $2 \mathrm{~min}$ and $95^{\circ} \mathrm{C}$ for $2 \mathrm{~min}$, followed by 40 cycles of amplification $\left(95^{\circ} \mathrm{C}\right.$ for $30 \mathrm{~s}, 57^{\circ} \mathrm{C}$ for $30 \mathrm{~s}$ and $72^{\circ} \mathrm{C}$ for $30 \mathrm{~s})$. The primers were validated using melting curve, amplification and standard curve analyses and notemplate control reactions. For the standard curve analysis, one DNA sample was serial diluted to $2.5,5,10$, 20,40 and $80 \mathrm{ng} / \mu \mathrm{l}$ and measured again using a spectrophotometer. Each concentration was analyzed in three-fold through qPCR to determine the amplification efficiency.

Each genomic DNA sample was diluted to $10 \mathrm{ng} / \mu \mathrm{l}$ in double-distilled water and assessed using qPCR in triplicate reactions. The qPCR was performed using the iCycler system (Bio-Rad, Hercules, CA), with individual PCR tubes (Bio-Rad, cat\# TLS0801, Hercules, CA). In the qPCR assay, the relative copy numbers were assigned after comparing the $\mathrm{Ct}$ values with the standard curve and the amount of copies in 1 ng of reference DNA (assumed as one unit).

\section{Additional files}

Additional file 1: Comparison of CNVs and CNVRs between galGal3 and galGal4 genome sequences.

Additional file 2: Comparison of the CNVRs identified in the present study with those reported in other studies.

Additional file 3: Information on the genes in the CNVRs identified in the present study.

Additional file 4: Gene ontology and pathway analyses of genes in the CNVRs identified in the present study.

Additional file 5: Previously reported QTLs that overlapped with the CNVRs identified in the present study.

Additional file 6: Quantitative PCR primers for the six CNVR loci examined in the present study. 


\section{Abbreviations}

aCGH: Array-based comparative genome hybridization; CNV: Copy number variation; CNVR: CNV region; Mb: Megabases; PCR: Polymerase chain reaction; qPCR: Quantitative PCR; SNP: Single nucleotide polymorphism;

QTLs: Quantitative trait loci; PCCA: Propionyl coenzyme A carboxylase; PRLR: Prolactin receptor; SORL1: Sortilin-related receptor; THRSP: Thyroid hormone responsive; SOCS2: Suppressor of cytokine signaling 2; RHACD8: CD8a molecule; XC: Xichuan Black-bone chicken; SK: Silkie chicken; LS: Lushi chicken; GS: Gushi chicken; HD: Houdan chicken; GO: Gene ontology; KEGG: Kyoto encyclopedia of genes and genomes.

\section{Competing interests}

The authors declare that they have no competing interests.

\section{Authors' contributions}

HR and YP performed experiments, analyzed data, and wrote the manuscript. KX and TY conceived and designed experiments. WD, ZZ and WL contributed to the sample collection, and performed the DNA isolation and qPCR analyses. LZ and JR managed the animals, collected the samples and discussed the manuscript. All authors read and approved the final manuscript.

\section{Acknowledgments}

The authors would like to thank Dr. Jiying Wang (Shandong Academy of Agricultural Sciences) for valuable discussion and assistance with computational statistics and data analysis. The authors would also like to thank Prof. Xiaojun Liu (Henan Agricultural University) and Paul Hsu (Penn State College of Medicine) for critically reading the manuscript. This study was funded through the National Natural Science Foundations of China (NO.31101707), the Earmarked Fund for Modern Agro-industry Technology Research System (NO.CARS-41-K04), the Science and Technology Cooperation Fund Projects of Henan Province of China (122106000046) and the Postdoctoral Science Foundation of Henan Province (2011031)

Received: 28 March 2014 Accepted: 16 October 2014

Published: 25 October 2014

\section{References}

1. Freeman $\mathrm{L}$, Perry GH, Feuk L, Redon R, McCarroll SA, Altshuler DM, Aburatani H, Jones KW, Tyler-Smith C, Hurles ME, Carter NP, Scherer SW, Lee C: Copy number variation: new insights in genome diversity. Genome Res 2006, 16(8):949-961.

2. Redon R, Ishikawa S, Fitch KR, Feuk L, Perry GH, Andrews TD, Fiegler $H_{\text {, }}$ Shapero MH, Carson AR, Chen W, Cho EK, Dallaire S, Freeman JL, Gonzalez JR, Gratacos M, Huang J, Kalaitzopoulos D, Komura D, MacDonald JR, Marshall CR, Mei R, Montgomery L, Nishimura K, Okamura K, Shen F, Somerville MJ, Tchinda J, Valsesia A, Woodwark C, Yang F, et al: Global variation in copy number in the human genome. Nature 2006 444(7118):444-454

3. Stankiewicz P, Lupski JR: Structural variation in the human genome and its role in disease. Annu Rev Med 2010, 61:437-455.

4. Mills RE, Walter K, Stewart C, Handsaker RE, Chen K, Alkan C, Abyzov A, Yoon SC, Ye K, Cheetham RK, Chinwalla A, Conrad DF, Fu Y, Grubert F Hajirasouliha I, Hormozdiari F, lakoucheva LM, lqbal Z, Kang S, Kidd JM Konkel MK, Korn J, Khurana E, Kural D, Lam HY, Leng J, Li R, Li Y, Lin CY, Luo $R$, et al: 1000 Genomes project: mapping copy number variation by population-scale genome sequencing. Nature 2011, 470(7332):59-65.

5. Pomp D, Nehrenberg D, Estrada-Smith D: Complex genetics of obesity in mouse models. Annu Rev Nutr 2008, 28:331-345.

6. McCarroll SA, Hadnott TN, Perry GH, Sabeti PC, Zody MC, Barrett JC, Dallaire S, Gabriel SB, Lee C, Daly MJ, Altshuler DM: International HapMap consortium: common deletion polymorphisms in the human genome. Nat Genet 2006, 38(1):86-92.

7. Hinds DA, Kloek AP, Jen M, Chen X, Frazer KA: Common deletions and SNPs are in linkage disequilibrium in the human genome. Nat Genet 2006, 38(1):82-85.

8. Bickhart DM, Hou Y, Schroeder SG, Alkan C, Cardone MF, Matukumalli LK, Song J, Schnabel RD, Ventura M, Taylor JF, Garcia JF, Van Tassell CP, Sonstegard TS, Eichler EE, Liu GE: Copy number variation of individual cattle genomes using next-generation sequencing. Genome Res 2012, 22(4):778-790
9. lafrate AJ, Feuk L, Rivera MN, Listewnik ML, Donahoe PK, Qi Y, Scherer SW, Lee C: Detection of large-scale variation in the human genome. Nat Genet 2004, 36(9):949-951.

10. Tuzun E, Sharp AJ, Bailey JA, Kaul R, Morrison VA, Pertz LM, Haugen E, Hayden $\mathrm{H}$, Albertson D, Pinkel D, Olson MV, Eichler EE: Fine-scale structural variation of the human genome. Nat Genet 2005, 37(7):727-732.

11. Conrad DF, Andrews TD, Carter NP, Hurles ME, Pritchard JK: A high-resolution survey of deletion polymorphism in the human genome. Nat Genet 2006, 38(1):75-81.

12. Sebat J, Lakshmi B, Troge J, Alexander J, Young J, Lundin P, Månér S, Massa H, Walker M, Chi M, Navin N, Lucito R, Healy J, Hicks J, Ye K, Reiner A, Gilliam TC, Trask B, Patterson N, Zetterberg A, Wigler M: Large-scale copy number polymorphism in the human genome. Science 2004, 305(5683):525-528.

13. Locke DP, Sharp AJ, McCarroll SA, McGrath SD, Newman TL, Cheng Z, Schwartz S, Albertson DG, Pinkel D, Altshuler DM, Eichler EE: Linkage disequilibrium and heritability of copy-number polymorphisms within duplicated regions of the human genome. Am J Hum Genet 2006, 79(2):275-290

14. Sharp AJ, Locke DP, McGrath SD, Cheng Z, Bailey JA, Vallente RU, Pertz LM Clark RA, Schwartz S, Segraves R, Oseroff W, Albertson DG, Pinkel D, Eichle EE: Segmental duplications and copy-number variation in the human genome. Am J Hum Genet 2005, 77(1):78-88.

15. Feuk $L$, Carson AR, Scherer SW: Structural variation in the human genome. Nat Rev Genet 2006, 7(2):85-97.

16. Liu GE, Hou Y, Zhu B, Cardone MF, Jiang L, Cellamare A, Mitra A, Alexander LJ, Coutinho LL, Dell'Aquila ME, Gasbarre LC, Lacalandra G, Li RW, Matukumalli LK, Nonneman D, Regitano LC, Smith TP, Song J, Sonstegard TS, Van Tassell CP, Ventura M, Eichler EE, McDaneld TG, Keele JW: Analysis of copy number variations among diverse cattle breeds. Genome Res 2010, 20(5):693-703.

17. Fadista J, Thomsen B, Holm L-E, Bendixen C: Copy number variation in the bovine genome. BMC Genomics 2010, 11(1):284.

18. Hou Y, Liu GE, Bickhart DM, Cardone MF, Wang K, Kim ES, Matukumalli LK, Ventura M, Song J, VanRaden PM, Sonstegard TS, Van Tassell CP: Genomic characteristics of cattle copy number variations. BMC Genomics 2011, 12(1):127.

19. Bae JS, Cheong HS, Kim HL, NamGung S, Park TJ, Chun JY, Kim J, Pasaje CF, Lee JS, Shin HD: Identification of copy number variations and common deletion polymorphisms in cattle. BMC Genomics 2010, 11(1):232.

20. Fontanesi PL, Beretti F, Martelli PL, Colombo M, Dall'Olio S, Occidente M, Portolano B, Casadio R, Matassino D, Russo V: A first comparative map of copy number variations in the sheep genome. Genomics 2011, 97(3):158-165.

21. Fontanesi PL, Martelli PL, Beretti F, Riggio V, Dall'Olio S, Colombo M, Casadio R, Russo V, Portolano B: An initial comparative map of copy number variations in the goat (Capra hircus) genome. BMC Genomics 2010, 11(1):639.

22. Ramayo-Caldas Y, Castelló A, Pena RN, Alves E, Mercadé A, Souza CA Fernández AJ, Perez-Enciso M, Folch JM: Copy number variation in the porcine genome inferred from a 60 k SNP BeadChip. BMC Genomics 2010 11(1):593.

23. Wang J, Jiang J, Fu W, Jiang L, Ding X, Liu JF, Zhang Q: A genome-wide detection of copy number variations using SNP genotyping arrays in swine. BMC Genomics 2012, 13(1):273

24. Li Y, Mei S, Zhang X, Peng X, Liu G, Tao H, Wu H, Jiang S, Xiong Y, Li F: Identification of genome-wide copy number variations among diverse pig breeds by array CGH. BMC Genomics 2012, 13(1):725.

25. Fadista J, Nygaard M, Holm L-E, Thomsen B, Bendixen C: A snapshot of CNVs in the pig genome. PLoS One 2008, 3(12):e3916.

26. Burt DW: Chicken genome: current status and future opportunities. Genome Res 2005, 15(12):1692-1698.

27. Skinner BM, Robertson LB, Tempest HG, Langley EJ, loannou D, Fowler KE, Crooijmans RP, Hall AD, Griffin DK, Völker M: Comparative genomics in chicken and Pekin duck using FISH mapping and microarray analysis. BMC Genomics 2009, 10(1):357.

28. Tian M, Wang Y, Gu X, Feng C, Fang S, Hu X, Li N: Copy number variants in locally raised Chinese chicken genomes determined using array comparative genomic hybridization. BMC Genomics 2013, 14(1):262

29. Völker M, Backström N, Skinner BM, Langley EJ, Bunzey SK, Ellegren H, Griffin DK: Copy number variation, chromosome rearrangement, and their association with recombination during avian evolution. Genome Res 2010, 20(4):503-511 
30. Jia X, Chen S, Zhou H, Li D, Liu W, Yang N: Copy number variations identified in the chicken using a 60 K SNP BeadChip. Anim Genet 2013, 44(3):276-284

31. Wang Y, Gu X, Feng C, Song C, Hu X, Li N: A genome-wide survey of copy number variation regions in various chicken breeds by array comparative genomic hybridization method. Anim Genet 2012, 43(3):282-289.

32. Wang X, Nahashon S, Feaster TK, Bohannon-Stewart A, Adefope N: An initial map of chromosomal segmental copy number variations in the chicken. BMC Genomics 2010, 11(1):351.

33. Crooijmans RP, Fife MS, Fitzgerald TW, Strickland S, Cheng HH, Kaiser $P$, Redon R, Groenen MA: Large scale variation in DNA copy number in chicken breeds. BMC Genomics 2013, 14(1):398.

34. Griffin DK, Robertson LB, Tempest HG, Vignal A, Fillon V, Crooijmans RP, Groenen MA, Deryusheva S, Gaginskaya E, Carré W, Waddington D, Talbot R, Völker M, Masabanda JS, Burt DW: Whole genome comparative studies between chicken and turkey and their implications for avian genome evolution. BMC Genomics 2008, 9(1):168.

35. Rubin C-J, Zody MC, Eriksson J, Meadows JR, Sherwood E, Webster MT, Jiang L, Ingman M, Sharpe T, Ka S, Hallböök F, Besnier F, Carlborg O, Bed'hom B, Tixier-Boichard M, Jensen P, Siegel P, Lindblad-Toh K, Andersson L: Whole-genome resequencing reveals loci under selection during chicken domestication. Nature 2010, 464(7288):587-591.

36. Elferink MG, Vallée AA, Jungerius AP, Crooijmans RP, Groenen MA: Partial duplication of the PRLR and SPEF2 genes at the late feathering locus in chicken. BMC Genomics 2008, 9(1):391

37. Wright D, Boije H, Meadows JR, Bed'hom B, Gourichon D, Vieaud A, Tixier-Boichard M, Rubin C-J, Imsland F, Hallböök F, Andersson L: Copy number variation in intron 1 of SOX5 causes the Pea-comb phenotype in chickens. PLoS Genet 2009, 5(6):e1000512.

38. Gunnarsson U, Kerje S, Bed'hom B, Sahlqvist AS, Ekwall O, Tixier-Boichard M, Kämpe $\mathrm{O}$, Andersson L: The Dark brown plumage color in chickens is caused by an $8.3-\mathrm{kb}$ deletion upstream of SOX10. Pigment Cell Melanoma Res 2011, 24(2):268-274.

39. Dorshorst B, Okimoto R, Ashwell C: Genomic regions associated with dermal hyperpigmentation, polydactyly and other morphological traits in the Silkie chicken. J Heredity 2010, 101(3):339-350.

40. Dorshorst B, Molin A-M, Rubin C-J, Johansson AM, Strömstedt L, Pham M-H, Chen C-F, Hallböök F, Ashwell C, Andersson L: A complex genomic rearrangement involving the endothelin 3 locus causes dermal hyperpigmentation in the chicken. PLOS Genet 2011, 7(12):e1002412.

41. Shinomiya A, Kayashima Y, Kinoshita K, Mizutani M, Namikawa T, Matsuda Y, Akiyama T: Gene duplication of endothelin 3 is closely correlated with the hyperpigmentation of the internal organs (Fibromelanosis) in Silky Chickens. Genetics 2012, 190(2):627-638.

42. Sugiyama T, Kumagai H, Morikawa Y, Wada Y, Sugiyama A, Yasuda K, Yokoi N, Tamura S, Kojima T, Nosaka T, Senba E, Kimura S, Kadowaki T, Kodama T, Kitamura T: A novel low-density lipoprotein receptor-related protein mediating cellular uptake of apolipoprotein E-enriched $\beta$-VLDL in vitro. Biochemistry 2000, 39(51):15817-15825.

43. Yang PK, Tian YD, Sun GR, Jiang RR, Han RL, Kang XT: Deposition rule of yolk cholesterol content in two different breeds. Genet Mol Res 2013, 12(4):5786-5792.

44. Gamazon ER, Nicolae DL, Cox NJ: A study of CNVs as trait-associated polymorphisms and as expression quantitative trait loci. PLOS Genet 2011, 7(2):e1001292

45. Huang DW, Sherman BT, Lempicki RA: Systematic and integrative analysis of large gene lists using DAVID bioinformatics resources. Nat Protoc 2009, 4(1):44-57.

46. Ashburner M, Ball CA, Blake JA, Botstein D, Butler H, Cherry JM, Davis AP, Dolinski K, Dwight SS, Eppig JT, Harris MA, Hill DP, Issel-Tarver L, Kasarskis A, Lewis S, Matese JC, Richardson JE, Ringwald M, Rubin GM, Sherlock G: Gene Ontology: tool for the unification of biology. Nat Genet 2000, 25(1):25-29.

doi:10.1186/1471-2164-15-934

Cite this article as: Han et al:: Identification and functional

characterization of copy number variations in diverse chicken breeds.

BMC Genomics 2014 15:934.

\section{Submit your next manuscript to BioMed Central and take full advantage of:}

- Convenient online submission

- Thorough peer review

- No space constraints or color figure charges

- Immediate publication on acceptance

- Inclusion in PubMed, CAS, Scopus and Google Scholar

- Research which is freely available for redistribution 